\title{
Future perspectives of aminoglycoside therapy
}

\author{
F. A. Waldrogel \\ Infectious Disease Division, Department of Medicine, Hôpital Cantonal Universitaire, \\ Geneva, Switzerland
}

\section{Introduction}

Since the early isolation by Waksman of streptomycin from Streptomyces griseus, the field of aminoglycoside research has expanded considerably through the development of a variety of closely related compounds with well-defined antibacterial activities. From a biochemical point of view, members of the aminoglycoside family consist of a similar backbone, i.e. two or more aminosugars joined via a glycosidic linkage to a hexose (or aminocyclitol) nucleus. The various compounds differ among themselves by the aminosugars attached to the aminocyclitol (streptidine or 2-deoxystreptamine) nucleus. Clinically important aminoglycoside antibiotics include streptomycin, neomycin, gentamicin, sissomicin, tobramycin, netilmicin and amikacin. Although these agents exhibit some differences in their antibacterial spectrum and clinical applications, their chemical similarities give them similar properties, such as absorption, distribution, excretion and toxicity. So while the host handles these related compounds in a similar way, bacteria behave differently towards them (Korzeniowski \& Hook, 1979; Waldvogel \& Acar, 1978).

Heterogeneous bacterial susceptibility towards aminoglycosides can be explained by at least three groups of mechanisms. Firstly, ribosomal alterations can modify the binding site on the $30 \mathrm{~s}$ ribosomal fractions for streptidine-containing, but not for 2-deoxystreptamine-containing aminoglycosides, thereby preventing the attachment of these aminoglycosides to their target (Hewitt, 1983). Secondly, changes in cell wall permeability, and/or in the transmembrane transport mechanisms may prevent the antibiotic from being carried against a gradient into the cytosol (Bryan, 1977). Lastly, and undoubtedly the best-known resistance mechanism, is enzymic, in which a variety of enzymes, some plasmid mediated, located within the periplasmic space, can neutralize the antibiotics in situ by means of adenylylating, acetylating, and phosphorylating mechanisms (Price et al., 1981). It is also conceivable that the intrinsic resistance of some bacterial species towards aminoglycosides is due to other and as yet unknown factors.

Before considering the future of the aminoglycoside antibiotics, I will briefly enumerate their major clinical uses, their advantages and disadvantages, as well as the problems encountered during their administration. Moreover, since $\beta$-lactam antibiotics have made such considerable progress in recent years to provide compounds with a similar antibiotic spectrum to aminoglycosides, I shall try to analyse with similar criteria their present indications, advantages and disadvantages. Finally, I shall try to delineate what improvements the clinician is expecting from future research in the field of aminoglycoside antibiotics to improve their present profile. 


\section{Clinical use of aminoglycosides}

It is generally accepted that there are at present seven major indications for treatment with aminoglycoside antibiotics, omitting the use of streptomycin in mycobacterial diseases.

(1) Severe infections due to Gram-negative aerobic bacilli. Limitations in this major indication include resistance to the aminoglycoside and susceptibility to one of the less toxic compounds, such as ampicillin and cephalosporins. Usually aminoglycosides are used to initiate therapy, but are often discontinued thereafter in favour of other antibiotics.

(2) In combination with an anti-pseudomonal $\beta$-lactam antibiotic for serious infections caused by Pseudomonas aeruginosa in patients with apparently normal defence mechanisms. Whether the $\beta$-lactam compound is an absolute requirement in non-neutropenic patients has been discussed in detail recently by Young (1982).

(3) Combined with a $\beta$-lactam antipseudomonal drug as empirical therapy in febrile, leukopenic compromised hosts (Lau et al., 1977; Keating et al., 1979; Love et al., 1979). Data from several laboratories and clinical studies have shown that the best results are expected if the antibiotic combination is synergistic in vitro (Schimpff et al., 1971; Klastersky et al., 1977). Poor results were obtained if the offending micro-organism was resistant towards one of the antibiotics used (Lau et al., 1977; Anderson et al., 1978), particularly in patients with ultimately fatal disease. Since antibiotic susceptibility of the nosocomial organisms often responsible for infections in compromised hosts varies greatly with time, clinical setting, and geography, it is imperative to choose a $\beta$-lactam antipseudomonal compound and an aminoglycoside which fulfil local sensitivity requirements.

(4) Systemic staphylococcal infections. Based on in vitro data (Watanakunakorn \& Glotzbecker, 1974) and some experimental studies (Steigbigel et al., 1975), several authors have suggested a combination of a $\beta$-lactamase-stable penicillin and an aminoglycoside to treat such systemic infections. A collaborative clinical study, however, has failed to show any significant benefit from such a combination, except in drug addicts, where combination therapy caused a more rapid clinical response without altering morbidity or mortality (Korzeniowski \& Sande, 1982). In other Staphylococcus aureus infections, aminoglycosides alone or in combination have never appeared to be more effective than $\beta$-lactam antibiotics.

(5) Enterococcal bloodstream infections and resulting endocarditis. Antibiotic combinations including penicillin $\mathrm{G}$ or vancomycin plus an aminoglycoside, such as gentamicin, have been synergistic against most enterococci and are now standard therapy.

(6) Infections following contaminated bowel and gynecological surgery can be prevented to a large extent by the use of an antibiotic regimen aimed at Gram-negative facultative aerobic bacilli and anaerobes. Thus, aminoglycosides combined with a drug effective against Bacteroides fragilis (for example, clindamycin, cefoxitin, or metronidazole), are highly effective, but other regimens with a similar antibacterial spectrum are equally active, as discussed recently in detail by Styrt \& Gorbach (1982).

(7) Prophylaxis in bowel surgery (Clarke et al., 1977; Bartlett et al., 1978), in cirrhotic patients with hepatic encephalopathy, and in immunocompromised, 
leukopenic patients (for review see Pizzo, 1983). Whether these indications are all justified from an epidemiological point of view remains debatable. Paromomycin has been used as a gastrointestinal disinfectant in shigellosis.

\section{Advantages and disadvantages of aminoglycosides}

The qualities of aminoglycoside antibiotics are self-evident. They have been in clinical use for many years already, 20 in the case of gentamicin, and are therefore well known to doctors. They cover an antibacterial spectrum for which there were few alternatives available until a few years ago. Rapid progress in the field of antibiotic assay systems has provided the clinician with the opportunity to follow serum levels during therapy, adjusting dosages if necessary. This has in turn led to the development of computer programs for adjusting aminoglycoside regimens with great accuracy by taking into consideration many host-specific variables (Appel \& Neu, 1978). Their great chemical stability in vitro permits easy storage, and the absence of metabolic transformation in the host permits their evaluation with simple pharmacokinetic models. Other advantages include their rapid bactericidal effect, and the absence of an inoculum effect for bacteria without drug-inactivating enzymes. Finally, their synergistic effect with $\beta$-lactam antibiotics against some Gram-negative bacilli, against many Ps. aeruginosa strains, and against enterococci is valuable.

Clinical use of aminoglycosides has raised, however, over the years, a variety of problems and questions which are still largely unsolved. First of all, as a reflection of their polar polycationic charge, they are not absorbed after oral administration. Their distribution within the host is limited, probably for similar reasons, their penetration into the cerebrospinal fluid, bile and lung secretions being poor, and their cellular penetration unpredictable or sometimes absent. Their biological disposition is altered by a variety of factors, including age, renal function, presence of ascites, fever, underlying diseases, and obesity. They can be inactivated under some conditions by carbenicillin and other $\beta$-lactam antibiotics (Noone \& Pattison, 1971; Farchione, 1981). Although probably of little clinical relevance except for patients in renal failure, this observation raises the question whether host-specific polyanions present in interstitial fluid and inflamed tissues might have similar effects. Changes in ionic composition, such as lowering of the $\mathrm{pH}$ or increasing $\mathrm{Ca}^{2+}$ and $\mathrm{Mg}^{2+}$ concentrations, greatly decrease the antibacterial activities of most aminoglycosides against $P s$. aeruginosa and some other organisms (Vaudaux, 1981). Reversible binding to lysed polymorphonuclear leukocytes, as found in exudates, can completely abolish their antibacterial effect (Vaudaux \& Waldvogel, 1980). They have a low therapeutic ratio, peak levels of $5 \mathrm{mg} / \mathrm{l}$ for gentamicin for instance being considered barely adequate, but $10 \mathrm{mg} / 1$ almost in the toxic range. Therefore, their two major toxicities, for the kidney and inner ear are still unsolved problems and limit their widespread use. Finally, emergence of resistance has become a familiar and major problem in many centres.

\section{Alternatives to aminoglycoside treatment}

During the last years, we have seen an explosive development in the field of $\beta$-lactam antibiotics, resulting in their use as alternative drugs in severe, Gram-negative infections. Third-generation cephalosporins are poorly active indeed against Gram-positive organisms, but encompass the same spectrum of antimicrobial activity as aminoglycosides such as gentamicin, tobramycin, netilmicin and amikacin (Hewitt, 1983). In addition, 
the variety of third-generation cephalosporins available gives them some specific additional advantages over aminoglycosides. Thus latamoxef (moxalactam) is also active against obligate anaerobes, and third-generation cephalosporins are often fully active against organisms resistant to earlier cephalosporins or aminoglycosides. Depending on the infecting organism(s), treatment can be tailored by choosing a cephalosporin characterized by broad- or narrow-spectrum activity. Furthermore, some compounds present interesting pharmacokinetic characteristics, such as high penetration into the bile (e.g. cefoperazone), or extremely prolonged elimination rates allowing single daily parenteral administration (e.g. ceftriaxone). Their diffusion into some body compartments can be particularly helpful, such as the good penetration of latamoxef (moxalactam) across inflamed meninges into the cerebrospinal fluid, which makes it a useful antibiotic in purulent meningitis (Schaad et al., 1981). Ototoxicity is absent and nephrotoxicity extremely rare (Hewitt, 1983). Finally, some of them act synergistically with aminoglycosides.

Putting all these advantages together, one wonders why third-generation cephalosporins have not completely replaced aminoglycosides yet for the treatment of Gramnegative infections. Indeed, their major therapeutic indications are similar to those mentioned for aminoglycosides, and include severe Gram-negative infections due to nosocomial organisms, such as bacteraemias, biliary tract infections and pneumonias, particularly if the offending organism has been identified. For instance, Serratia spp. are particularly susceptible to cefotaxime, and Ps. aeruginosa to cefsulodin. In addition, latamoxef (moxalactam) has become a valuable member of the antibiotic armamentarium for the treatment of purulent meningitis due to ampicillin-resistant Enterobacteriaceae or to ampicillin-resistant Haemophilus influenzae (Schaad et al., 1981). Other important indications for third-generation cephalosporins include infections due to aminoglycosideresistant Gram-negative organisms, and severe Gram-negative infections in patients with renal insufficiency (Neu, 1982). Finally, they have also been used as empirical therapy in febrile compromised hosts, most often in association with aminoglycosides (Hewitt, 1983).

In spite of their recent success, use of third-generation cephalosporins is not without problems. Although the experience accumulated with these compounds extends only over a few years, their efficacy has already been shown to vary with location and time, and clearly reflects epidemiological changes in antibiotic susceptibilities (Neu, 1982). Some cephalosporins are metabolized in the host, cefotaxime being for instance de-acetylated in the liver. In vitro they occasionally show inoculum effects, and resistant variants can be easily obtained in the laboratory. Their bactericidal activity is poor against Grampositive organisms, and is virtually absent against enterococci which have also sometimes emerged as secondary invaders (Moellering, 1982). Finally, they have been held responsible for a variety of adverse effects, such as hypersensitivity reactions, leukopenias, platelet dysfunction, disulfiram effects and superinfections with Candida species and other secondary invaders (Neu, 1982).

\section{The future of aminoglycoside antibiotics}

Although some of the newer $\beta$-lactam compounds, including monobactam antibiotics and some quinolone derivatives, will definitely offer future alternatives to aminoglycoside therapy, it is unlikely that they will supplant it completely because of their own specific problems. Nevertheless, the question can be asked how aminoglycosides could be further improved to keep up with the rapid developmeni of newer compounds. Firstly, the 
further development of less nephro- and ototoxic compounds would seem particularly advisable, although from the data presently available I doubt whether within this family toxicity can be completely prevented (Lerner et al., 1983). Co-administration of other agents protecting the renal function, such as divalent cations, has been recently suggested by new experimental data (Humes et al., 1983; Elliot et al., 1982). The discovery or synthesis of non-toxic, non-aminoglycosidic inhibitors of inactivating enzymes would increase their longevity by allowing their use even against some resistant organisms. Other areas of research should include the development of new pharmacological preparations of aminoglycosides with better diffusing capacities, such as increased diffusion across the meninges, into inflammatory foci and possibly into phagocytes. In this respect, the suggestions of packaging aminoglycosides into liposomes or of linking them covalently to carrier molecules warrants careful experimental evaluation. Finally, efforts should be made towards a better understanding of the bacterial oxygen-dependent, aminoglycosideuptake mechanism (Bryan, 1977), and its possible stimulation by non-toxic molecules. It is conceivable that an induction of these mechanisms may allow aminoglycosides to express their lethal intracellular effect at much smaller extracellular concentrations, thus reducing potential nephro- or ototoxicity. Developments in these fields of aminoglycoside research would ensure them a future similar to that presently seen with the $\beta$-lactam compounds.

\section{References}

Anderson, E. T., Young, L. S. \& Hewitt, W. L. (1978). Antimicrobial synergism in the therapy of Gram-negative rod bacteremia. Chemotherapy 24, 45.

Appel, G. B. \& Neu, H. C. (1978). Gentamicin in 1978. Annals of Internal Medicine 89, 528-38.

Bartlett, J. G., Condon, R. E., Gorbach, S. L., Clarke, J. S., Nichols, R. L. \& Ochi, S. (1978). Veterans Administration cooperative study on bowel preparation for elective colorectal operations: impact of oral antibiotic regimen on colonic flora, would irrigation cultures and bacteriology of septic complications. Annals of Surgery 188, 249-54.

Bryan, L. E. (1977). Effects of membrane-energy mutations and cations on streptomycin and gentamicin accumulation by bacteria: a model for entry of streptomycin and gentamicin in susceptible and resistant bacteria. Antimicrobial Agents and Chemotherapy 12, 163-77.

Clarke, J. S., Condon, R. E., Bartlett, J. G., Gorbach, S. L., Nichols, R. L. \& Ochi, S. (1977). Preoperative oral antibiotics reduce septic complications of colon operations: results of prospective, randomized double-blind clinical study. Annals of Surgery 186, 251-9.

Elliot, W. C., Gilbert, D. N., Defehr, J., Bennett, W. M. \& MacCarron., D. A. (1982). Protection from experimental gentamicin toxicity by dietary calcium loading. Kidney International 21, 216.

Farchione, L. A. (1981). Inactivation of aminoglycosides by penicillins. Journal of Antimicrobial Chemotherapy 8 (Suppl. A), 27-36.

Hewitt, W. L. (1983). The third generation cephalosporins. In Current Clinical Topics in Infectious Diseases (Remington, J. S. \& Swartz, M. N., Eds), pp. 403-23. McGraw-Hill, New York.

Humes, H. D., Sastrasinh, M. \& Weinberg, J. M. (1983). Is calcium a competitive inhibitor of the gentamicin renal membrane interaction which ameliorates gentamicin nephrotoxicity? Kidney International 23, 204.

Keating, M. J., Bodey, G. P., Valdivies, M. \& Rodriguez, V. (1979). A randomized comparative trial of three aminoglycosides, comparison of continuous infusions of gentamicin, amikacin and sisomicin combined with carbenicillin in the treatment of infections in neutropenic patients with malignancies. Medicine 58, 159-70.

Klastersky, J., Meunier-Carpentier, F. \& Prevost, J. M. (1977). Significance of antibiotic synergism for the outcome of Gram negative sepsis. American Journal of Medical Science $273,157-67$.

Korzeniowski, O. \& Hook, E. W. (1979). Aminocyclitols: aminoglycosides and spectinomycin. In Principles and Practice of Infectious Diseases (Mandell, G. L., Douglas, R. G. \& Bennett, J. E., Eds), pp. 249-73. John Wiley \& Sons, New York.

Korzeniowski, O. \& Sande, M. A. (1982). Combination antimicrobial therapy for Staphylococcus aureus endocarditis in patients addicted to parenteral drugs and in nonaddicts. Annals of Internal Medicine 97, 496-503. 
Lau, W. K., Young, L. S., Black, R. E., Winston, D. J., Linne, S. R., Weinstein, R. J. \& Hewitt, W. L. (1977). Comparative efficacy and toxicity of amikacin/carbenicillin versus gentamicin/ carbenicillin in leukopenic patients. A randomized prospective trial. American Journal of Medicine 62, 959-66.

Lerner, A. M., Reyes, M. P., Cone, L. A., Blair, D. C., Jansen, W., Wright, G. \& Lorber, R. R. (1983). Randomised, controlled trial of the comparative efficacy, auditory toxicity, and nephrotoxicity of tobramycin and netilmicin. Lancet $i, 1123-6$.

Love, L. J., Schimpff, S. C., Hahn, D. M., Young, V. M., Standford, H. C., Bender, J. F., Fortner, C. L. \& Wiernik, P. H. (1979). Randomized trial of empiric therapy with ticarcillin in combination with gentamicin, amikacin, or netilmicin in febrile patients with granulocytopenia and cancer. American Journal of Medicine 66, 603-10.

Moellering, Jr, R. C. (1982). Enterococcal infections in patients with moxalactam. Reviews of Infectious Diseases 4, S708-11.

Murray, B. E. \& Moellering, Jr, R. C. (1978). Patterns and mechanisms of antibiotic resistance. Medical Clinics of North America 50, 899-923.

Neu, H. C. (1982). The new beta-lactamase-stable cephalosporins. Annals of Internal Medicine 97, 408-19.

Noone, P. \& Pattison, J. R. (1971). Therapeutic implications of interaction of gentamicin and penicillins. Lancet ii 2, 575-8.

Pizzo, P. A. (1983). Antimicrobial prophylaxis in the immunosupressed cancer patient. In Current Clinical Topics in Infectious Diseases (Remington, J. S. \& Swartz, M. N., Eds), pp. 153-85. McGraw-Hill Book Company, New York.

Price, K. E., Kresel, P. A., Farchione, L. A., Siskin, S. B. \& Karpow, S. A. (1981). Epidemiological studies of aminoglycoside resistance in the U.S.A. Journal of Antimicrobial Chemotherapy 8 , (Suppl. A), 89-105.

Schaad, U. B., McCracken, G. H., Threlkeld, N. \& Thomas, M. L. (1981). Clinical evaluation of a new broad-spectrum oxa-beta-lactam antibiotic, moxalactam, in neonates and infants. Journal of Pediatrics 98, 129-36.

Schimpff, S., Satterlee, W., Young, V. M. \& Serpick, A. (1971). Empiric therapy with carbenicillin and gentamicin for febrile patients with cancer and granulocytopenia. New England Journal of Medicine 284, 1061-5.

Steigbigel, R. T., Greenmann, L. \& Remington, J. S. (1975). Antibiotic combinations in the treatment of experimental Staphylococcus aureus infection. Journal of Infectious Diseases 131, 245-51.

Styrt, B. \& Gorbach, S. L. (1982). Prophylactic antibiotics for bowel surgery. In Current Clinical Topics in Infectious Diseases (Remington, J. S. \& Schwartz, M. N., Eds), pp. 286-308. McGraw'Hill, New York.

Vaudaux, P.\& Waldvogel, F. A. (1980). Gentamicin inactivation in purulent exudates: role of cell lysis. Journal of Infectious Diseases 142, 586-93.

Vaudaux, P. (1981). Peripheral inactivation of gentamicin. Journal of Antimicrabial Chemotherapy 8, (Suppl. A), 17-25.

Waldvogel, F. A. \& Acar, J. (1978). Médicaments antibactériens. In Thérapeutique médicale (Fabre, J., Ed.), pp. 131-62. Flammarion, Paris.

Watanakunakom, G. \& Glotzbecker, C. (1974). Enhancement of the effects of antistaphylococcal antibiotics by aminoglycosides. Antimicrobial Agents and Chemotherapy 6, 802-6.

Young, L. S. (1982). Combination or single drug therapy for Gram-negative sepsis. In Current Clinical Topics in Infectious Diseases (Remington, J. S. \& Swartz, M. N., Eds), pp. 177-205. McGraw-Hill, New York. 